Bundesgesundheitsbl 2010 · 53:91-102

DOI 10.1007/s00103-009-1016-0

Online publiziert: 27. Januar 2010

(c) Springer-Verlag 2010

C. Kröger ${ }^{1}$ U. Mons ${ }^{2} \cdot$ G. Klärs ${ }^{3} \cdot$ B. Orth ${ }^{4} \cdot$ U. Maschewsky-Schneider ${ }^{5} \cdot$ T. Lampert $^{6}$

${ }^{1}$ IFT-Institut für Therapieforschung, München

${ }^{2}$ Deutsches Krebsforschungszentrum (DKFZ), Heidelberg

${ }^{3}$ Gesellschaft für Versicherungswissenschaft und -gestaltung (GVG), Köln

${ }^{4}$ Bundeszentrale für gesundheitliche Aufklärung (BZgA), Köln

${ }^{5}$ Berlin School of Public Health an der Charité, Charité - Universitätsmedizin Berlin

${ }^{6}$ Robert Koch-Institut (RKI), Berlin

\title{
Evaluation des Gesundheitsziels „Tabakkonsum reduzieren“
}

konsum nur die Kombination aus verhaltens- und verhältnisbezogenen Maßnahmen zum gewünschten Erfolg führt („Policy-Mix“). Bei der Entwicklung des Gesundheitsziels „Tabakkonsum reduzieren" einigten sich die Akteure in der entsprechenden $\mathrm{AG}^{1}$ auf ein Szenario, das eine Kombination der Maßnahmen enthält, die eine wissenschaftlich gesicherte Evidenz aufweisen [3].

Da diese umfassende Strategie nur in Teilschritten umsetzbar ist, wurden Teilziele und Maßnahmen entwickelt, die als Zwischenschritte und Indikatoren für eine Entwicklung in die gewünschte Richtung „Tabakkonsum reduzieren“ dienen.

Die Maßnahmen zur Veränderung von Verhaltensweisen (Verhaltensprävention) und zur Veränderung struktureller Rahmenbedingungen beziehungsweise Durchführung gesetzgeberischer Maßnahmen (Verhältnisprävention) können drei Zielbereichen zugeordnet werden:

- allgemeine Tabakentwöhnung (Ausstieg erleichtern),

- Förderung des Nichtrauchens insbesondere bei Kindern und Jugendlichen (Einstieg verhindern),

- Schutz vor Passivrauchen.

Um die Umsetzung des Gesundheitsziels zu unterstützen, verabschiedete der Ausschuss von gesundheitsziele.de am 6. Juli 2004 fünf Basismaßnahmen aus dem

\footnotetext{
1 Vorsitz: Prof. Dr. E. Pott (BZgA).
}

Spektrum umfassender Tabakkontrollpolitik:

1. Tabaksteuererhöhungen,

2. vollständiges Verbot direkter und indirekter Tabakwerbung,

3. Schutz vor Passivrauchen,

4. Förderung des Ausstiegs aus der abakabhängigkeit,

5. Maßnahmen zur Verhinderung des Einstiegs in das Rauchen.

Neben der Begründung und erwarteten Wirkung der einzelnen Maßnahmen wurden die für die jeweiligen Umsetzungsstrategien relevanten Akteure und Indikatoren für die Erfolgs- und Qualitätskontrolle beschrieben. Verbunden mit der Veröffentlichung der Basismaßnahmen war die Empfehlung an die Bundesregierung, auf dieser Grundlage einen „Nationalen Aktionsplan zur Verringerung des Tabakkonsums" zu entwickeln, der jedoch im Sinne einer umfassenden Tabakkontrolle um weitere Elemente auf der Verhaltens- und Verhältnisebene vervollständigt werden sollte [4]. Im Jahr 2008 legte der Drogen- und Suchtrat der Drogenbeauftragten Empfehlungen für einen solchen „Nationalen Aktionsplan zur Tabakprävention" vor [5].

Da Fortschritts- und Wirksamkeitsmessung notwendiger Bestandteil eines Gesundheitszieleprozesses ist, hat der hierfür zuständige Evaluationsbeirat von gesundheitsziele.de im Mai 2005 ein Evaluationskonzept vorgelegt. Eine Unterar- 


\begin{tabular}{|c|c|c|c|c|c|c|c|c|c|c|c|c|c|c|c|c|}
\hline & 1993 & 1994 & 1995 & 1996 & 1997 & 1998 & 1999 & 2000 & 2001 & 2002 & 2003 & 2004 & 2005 & 2006 & 2007 & 2008 \\
\hline \multicolumn{17}{|l|}{$\begin{array}{l}\text { Drogenaffinitäts- und } \\
\text { Rauchfrei-Studien }^{a}\end{array}$} \\
\hline $\begin{array}{l}\text { Männliche Jugendliche } \\
\text { (12 bis } 17 \text { Jahre) }\end{array}$ & 20,8 & - & - & - & 27,4 & - & - & - & 27,2 & - & 21,9 & 24,2 & 20,7 & - & 17,8 & 14,7 \\
\hline $\begin{array}{l}\text { Weibliche Jugendliche } \\
\text { (12 bis } 17 \text { Jahre) }\end{array}$ & 20,1 & - & - & - & 28,9 & - & - & - & 27,9 & - & 23,2 & 22,7 & 19,3 & - & 17,5 & 16,2 \\
\hline \multicolumn{17}{|l|}{ ESPAD-Studien ${ }^{b}$} \\
\hline $\begin{array}{l}\text { Schüler in 9. und } \\
\text { 10. Klassen }\end{array}$ & - & - & - & - & - & - & - & - & - & - & 44,9 & - & - & 35,2 & - & - \\
\hline $\begin{array}{l}\text { Schülerinnen in 9. und } \\
\text { 10. Klassen }\end{array}$ & - & - & - & - & - & - & - & - & - & - & 48,4 & - & - & 37,6 & - & - \\
\hline \multicolumn{17}{|l|}{ HBSC-Studien ${ }^{c}$} \\
\hline 15-jährige Schüler & - & 20,9 & - & - & - & 27,4 & - & - & - & 31,8 & - & - & - & 18,0 & - & - \\
\hline 15-jährige Schülerinnen & - & 28,8 & - & - & - & 33,0 & - & - & - & 31,9 & - & - & - & 22,3 & - & - \\
\hline \multicolumn{17}{|l|}{$\begin{array}{l}\text { Drogenaffinitäts- und } \\
\text { Rauchfrei-Studien }\end{array}$} \\
\hline $\begin{array}{l}\text { Junge Männer ( } 18 \text { bis } \\
25 \text { Jahre) }\end{array}$ & 51,0 & - & - & - & 53,7 & - & - & - & 46,7 & - & - & 44,8 & - & - & - & 42,2 \\
\hline $\begin{array}{l}\text { Junge Frauen ( } 18 \text { bis } \\
25 \text { Jahre) }\end{array}$ & 43,9 & - & - & - & 46,8 & - & - & - & 42,2 & - & - & 44,1 & - & - & - & 43,8 \\
\hline \multicolumn{17}{|l|}{$\begin{array}{l}\text { Epidemiologischer } \\
\text { Suchtsurvey }{ }^{d}\end{array}$} \\
\hline $\begin{array}{l}\text { Junge Männer ( } 18 \text { bis } \\
24 \text { Jahre) }\end{array}$ & - & - & 50,1 & - & 45,7 & - & - & 45,3 & - & - & 42,0 & - & - & 41,5 & - & - \\
\hline $\begin{array}{l}\text { Junge Frauen ( } 18 \text { bis } \\
24 \text { Jahre) }\end{array}$ & - & - & 33,8 & - & 36,6 & - & - & 39,5 & - & - & 39,7 & - & - & 37,8 & - & - \\
\hline \multicolumn{17}{|l|}{$\begin{array}{l}\text { Epidemiologischer } \\
\text { Suchtsurvey }^{d}\end{array}$} \\
\hline Männer (18 bis 59 Jahre) & - & - & 42,8 & - & 43,4 & - & - & 39,0 & - & - & 37,1 & - & - & 37,3 & - & - \\
\hline Frauen (18 bis 59 Jahre) & - & - & 29,3 & - & 30,2 & - & - & 30,6 & - & - & 30,5 & - & - & 28,8 & - & - \\
\hline \multicolumn{17}{|l|}{$\begin{array}{l}\text { Gesundheitssurveys des } \\
\text { RKI }^{d}\end{array}$} \\
\hline Männer (25 bis 69 Jahre) & - & - & - & - & - & 37,6 & - & - & - & - & 37,9 & - & - & 35,5 & - & - \\
\hline Frauen ( 25 bis 69 Jahre) & - & - & - & - & - & 28,8 & - & - & - & - & 31,1 & - & - & 27,8 & - & - \\
\hline
\end{tabular}

beitsgruppe ${ }^{2}$ entwickelte Erfolgsindikatoren der einzelnen Basismaßnahmen [6].

Die vorliegende Publikation beschreibt die Umsetzung des Evaluationskonzepts und berichtet über den Stand der Zielerreichung zu den fünf Basismaßnahmen.

\footnotetext{
2 Mitglieder der Unterarbeitsgruppe waren Vertreter/innen des IFT Institut für Therapieforschung, des Robert Koch-Instituts, des Deutschen Krebsforschungszentrums, der Technischen Universität Berlin und der Bundeszentrale für gesundheitliche Aufklärung.
}

\section{Datenquellen}

Zur Prävalenz des Rauchens in Deutschland liegen folgende wiederholte Erhebungen vor.

Für Erwachsene:

- Gesundheitssurveys des Robert Koch-Instituts (RKI) [7],

- Epidemiologische Suchtsurveys (ESA) des IFT Institut für Therapieforschung [8].

Für Jugendliche und junge Erwachsene:

- Drogenaffinitätsstudien (DAS) und Jugendstudien „Förderung des Nichtrauchens" der Bundeszentrale für gesundheitliche Aufklärung (BZgA) [9, $10,11,12]$,
- Europäische Schülerstudie zu Alkohol und anderen Drogen (ESPAD) des IFT [13] (Schülerinnen und Schüler aus 9. und 10. Klassen in sechs Bundesländern),

- Health Behaviour in School-aged Children (HBSC)-Studie [14] (Schülerinnen und Schüler aus 5., 7. und 9. Klassen),

- Kinder- und Jugendgesundheitssurvey (KiGGS) des RKI (bisher erst eine Erhebung von 2003 bis 2006, weitere Erhebungen sind geplant [15].

Neben den epidemiologischen Daten werden für die Evaluation der verschiedenen Basismaßnahmen weitere spezifische Datenquellen ausgewertet. 


\section{Aktuelle Prävalenzzahlen}

Die $\bullet$ Tab. 1 zeigt die Prävalenz der Raucher/innen in verschiedenen Repräsentativbefragungen von 1993 bis 2008. Die Daten der Jugendstudien „Förderung des Nichtrauchens" und der Drogenaffinitätsstudien der BZgA zeigen, dass sich die Prävalenz des Rauchens (Selbsteinschätzung als gelegentliche/r oder ständige/ $\mathrm{r}$ Raucher/in) bei den zwölf- bis 17-jährigen Jugendlichen von 28\% im Jahr 2001 auf 15\% im Jahr 2008 reduziert hat. Diese Reduktion ist sowohl bei weiblichen als auch männlichen Jugendlichen zu finden. Eine ähnliche Entwicklung lässt sich mit den Daten der HBSC-Studie für Nordrhein-Westfalen nachzeichnen [9].

Laut ESPAD-Studie ist bei Schüler(inne)n der 9. und 10. Klassen die 30-Tage-Prävalenz des Tabakkonsums von $46,7 \%$ im Jahr 2003 auf 36,5\% im Jahr 2007 zurückgegangen. Der Rückgang ist sowohl bei Schülerinnen (2003: 48,4\%; 2007: 37,6\%) als auch Schülern (2003: 44,9\%; 2007: 35,2\%) zu beobachten [13].

Bei jungen Erwachsenen im Alter von 18 bis 25 Jahren zeigen die Ergebnisse der Drogenaffinitätsstudie seit 2001 keine signifikante Änderung der Prävalenz des Rauchens. Insgesamt hat sich die Quote von $44,5 \%$ im Jahr 2001 auf $43,1 \%$ im Jahr 2008 nur unwesentlich verändert [9]. Auch im Epidemiologischen Suchtsurvey finden sich bei jungen Erwachsenen (18 bis 24 Jahre) von 2000 (42,5\%) bis 2006 $(39,7 \%)$ keine statistisch bedeutsamen Veränderungen der 30-Tage-Prävalenz des Rauchens. Bei den 18- bis 59-jährigen Erwachsenen hat seit dem Jahr 2000 keine Reduktion des Anteils rauchender Personen stattgefunden [8]. In den Gesundheitssurveys des RKI zeigt sich für die 25- bis 69-jährige Bevölkerung ein leichter Rückgang der Raucher/innen; bei den Männern von 37,9\% im Jahr 2003 auf 35,5\% im Jahr 2006, bei den 25- bis 69-jährigen Frauen von $31,1 \%$ auf $27,8 \%$ [16].

\section{Basismaßnahme 1: Tabaksteuererhöhungen}

Tabaksteuererhöhungen sollten möglichst kontinuierlich erfolgen und alle Tabakwaren betreffen [17]. Es wird erwartet, dass eine Erhöhung der Tabaksteuer zu einem

Bundesgesundheitsbl 2010 ·53:91-102 DOI 10.1007/s00103-009-1016-0

C) Springer-Verlag 2010

\section{Kröger · U. Mons · G. Klärs · B. Orth · U. Maschewsky-Schneider · T. Lampert Evaluation des Gesundheitsziels „Tabakkonsum reduzieren“}

\section{Zusammenfassung}

Der Kooperationsverbund gesundheitsziele. de hat Ziele und Maßnahmen definiert, um den Tabakkonsum in der Bevölkerung zu reduzieren. Fünf Basismaßnahmen wurden definiert: (1) Tabaksteuererhöhungen, (2) vollständiges Verbot direkter und indirekter Tabakwerbung, (3) Schutz vor Passivrauchen, (4) Förderung des Ausstiegs aus der Tabakabhängigkeit und (5) Maßnahmen zur Verhinderung des Einstiegs in das Rauchen. Die in den letzten Jahren erreichten Ergebnisse werden unter Zuhilfenahme der Daten verschiedener epidemiologischer Studien und anderer verfügbarer Statistiken zusammengefasst. (1) Tabaksteuererhöhungen hatten eine deutliche Erhöhung des Zigarettenpreises zur Folge. (2) Ein vollständiges Verbot der Tabakwerbung ist bislang nicht umgesetzt worden. Es zeigt sich eine Verlagerung der Werbeausgaben der Tabakindustrie in Bereiche, in denen Werbung weiterhin erlaubt ist. (3) Zum Schutz vor Passivrauchen wurden viele Maßnahmen umgesetzt. In der Bevölkerung zeigen sich ein zunehmendes Problembewusstsein, eine zunehmende Akzeptanz von Rauchverboten und ein Rückgang der Passivrauchbelastung. (4) Das Angebot von und die Nachfrage nach Entwöhnungsmaßnahmen sind gestiegen. (5) Erfolge der Maßnahmen zur Verhinderung des Einstiegs zeigen sich in einer sinkenden Raucherquote bei Jugendlichen, einer steigenden Nieraucherquote und in einer zunehmend kritischeren Einstellung der Nieraucher/innen gegenüber dem Rauchen. Es wird empfohlen, die begonnenen Anstrengungen weiter fortzuführen, da das Potenzial insbesondere an strukturellen, aber auch massenmedialen und verhaltensbezogenen Maßnahmen nicht ausgeschöpft ist.

Schlüsselwörter Gesundheitsziel · Rauchen · Passivrauchen . Tabakentwöhnung · Prävention

\section{Assessing the health target "Reduce tobacco consumption"}

\section{Abstract}

The consortium gesundheitsziele. de has defined health targets and actions to reduce tobacco consumption of the general population. Five primary fields of action were defined: (1) tax increase, (2) ban of advertisements, (3) protection against second-hand smoking, (SHS) (4) support of smoking cessation, and (5) actions to avoid taking up smoking. The achievements in recent years are described by means of epidemiological studies and other specific data. (1) Tax increases led to a significant rise of cigarette prices. (2) A complete ban of advertisements has not yet been achieved. The tobacco industry shifted expenditures to fields where advertising is still allowed. (3) Many actions to foster protection against SHS could be implemented. Public awareness has been raised, the ac- ceptance of smoking bans has grown, and exposure to SHS has been reduced. (4) Demand and supply of smoking cessation interventions have been enhanced. (5) Actions to avoid taking up smoking seem to be successful as the rate of smokers among young people has decreased, the number of never-smokers has increased, and a more critical attitude of non-smokers towards smoking could be observed. It is recommended that the above actions be continued because the capabilities of the structural, mass media, and behavioral interventions have not been fully exhausted.

\section{Keywords}

Health targets · Smoking $\cdot$ Second hand smoke $\cdot$ Smoking cessation $\cdot$ Prevention 
Rückgang des Rauchens besonders bei Jugendlichen und Kindern sowie in sozial benachteiligten Bevölkerungsgruppen beiträgt. Tabaksteuererhöhungen sollen sich unmittelbar auf die Nachfrage nach Tabakprodukten auswirken und die Zahl der Personen, die mit dem Rauchen aufhören, erhöhen, die Zahl derjenigen, die mit dem Rauchen anfangen, senken sowie bei verbleibenden Raucher(inne)n zu einer Reduktion des Rauchens führen [6].

Indikatoren zur Messung der Umsetzung und des Erfolgs der Maßnahme sind die Gesetzgebung (Tabaksteuergesetz, TabStG; Gesetz zur Änderung des Tabaksteuergesetzes und anderer Verbrauchsteuergesetze; Verordnung zur Durchführung des Tabaksteuergesetzes, TabStV), die Preisgestaltung, die auf Grundlage der Statistiken des Statistischen Bundesamtes von Lampert und List [16] aufbereitet wurden, sowie die Entwicklung der Prävalenz. Darüber hinaus liegen aus Repräsentativbefragungen Daten zur Akzeptanz und zu Verhaltensänderungen aufgrund von Tabaksteuererhöhungen vor $[6,18,19,20,21]$.

\section{Ergebnisse}

Im Dezember 2001 und Dezember 2003 sind zwei Gesetzesänderungen des Tabaksteuergesetzes (TabStG) in Kraft getreten, die in fünf Stufen vom 1. Januar 2002 bis 1 . September 2005 wiederholte Tabaksteuererhöhungen von einem bis 1,2 Cent pro Zigarette regeln [19, 20, 21, 22]. Seit 2005 ist keine Steuererhöhung mehr erfolgt. Die Steuererhöhungen wurden in verschiedenem Umfang, teilweise mit einer Erhöhung der Abgabepreise, an die Konsument(inn)en weitergegeben. Bei einem durchschnittlichen Zigarettenpreis von 13,89 Cent im Jahr 2001 gegenüber 21,85 Cent im Jahr 2007 ist ein Preiszuwachs von $57 \%$ festzustellen [16] (eigene Berechnung). Feinschnitt wird weniger stark besteuert als Fertigzigaretten.

Bevölkerungsbefragungen nach den einzelnen Stufen der Steuererhöhungen deuten auf eine zunehmende Akzeptanz von Tabaksteuererhöhungen hin [21]. Der Anteil der Personen, die die Tabaksteuererhöhung befürworten, betrug 33,7\% (Januar 2002), 35,5\% (Januar 2003), 43,0\%
(März 2004) beziehungsweise 41,6\% (Dezember 2004). Ein bedeutender Anteil an Personen berichtet, infolge der Steuererhöhungen ihr Rauchverhalten geändert zu haben $[19,20,21]$. Dies stimmt mit Befunden des Telefonischen Gesundheitssurveys 2005 des RKI überein. Die Preiserhöhungen haben bei $24 \%$ der Raucher und 22\% der Raucherinnen dazu geführt, dass sie weniger rauchen als früher. Auf eine andere Marke oder andere Tabakwaren sind $27 \%$ der Raucher und $32 \%$ der Raucherinnen umgestiegen. Sowohl die Reduktion als auch der Markenwechsel sind einkommensabhängig und bei Personen mit niedrigem Einkommen am häufigsten zu finden [7]. In den Studien der BZgA lässt sich nach den Preiserhöhungen ein Anstieg der rauchenden Zwölf- bis 25-Jährigen nachweisen, die vorwiegend selbst gedrehte Zigaretten rauchen [9].

\section{Basismaßnahme 2: Vollständiges Verbot direkter und indirekter Tabakwerbung}

Tabakwerbung erhöht nachweislich die Nachfrage nach Tabakprodukten [23, 24], erzeugt ein positives gesellschaftliches Klima für den Tabakkonsum und setzt das Rauchen in Zusammenhang mit Lebensstilen und Erlebniswerten. Dies gilt sowohl für die direkte Bewerbung von Tabakprodukten als auch für indirekte Formen der Werbung, zum Beispiel das Markensponsoring. Wie bei Tabaksteuererhöhungen ist davon auszugehen, dass ein Verbot von Tabakwerbung sowohl das Einstiegs- als auch das Ausstiegsverhalten positiv beeinflusst und bei verbleibenden Raucher(inne)n einen geringeren Konsum bewirkt [25]. Weiterhin hat die nicht markenbezogene Darstellung von Rauchszenen in Fernseh- und Kinoproduktionen eine Wirkung auf Kinder und Jugendliche. Diese haben ein doppelt so hohes Risiko, mit dem Rauchen zu beginnen, wenn in von ihnen gesehenen Filmen häufig geraucht wird. Im internationalen Vergleich wird in deutschen Serien und Spielfilmen deutscher Produktionen doppelt so häufig geraucht wie in ausländischen Produktionen. Dies betrifft auch Filme mit einer Altersfreigabe für Kinder [26].
Als Erfolgs- und Messindikatoren für das vollständige Verbot direkter und indirekter Werbung für Tabakerzeugnisse ${ }^{3}$ wurden im Rahmen des Evaluationskonzeptes [27] formuliert: (1) Verringerung der Werbe- und Sponsoringausgaben der Tabakindustrie durch eine Senkung der Werbeträger-bezogenen Gesamtausgaben der Tabakindustrie und (2) Bestehen eines umfassenden Tabakwerbeverbotes durch gesetzliche Vorschriften zur Regelung der Tabakwerbung und freiwilliger Selbstbeschränkungsvereinbarungen mit der Tabakindustrie.

\section{Ergebnisse}

\section{Verringerung der Werbe- und Sponsoringausgaben}

Der jährliche Drogen- und Suchtbericht der Drogenbeauftragten enthält Angaben zu Werbeausgaben der Tabakindustrie, die auf Grundlage des Artikels 13 der Tabakrahmenkonvention [28] von der Tabakindustrie nach Werbeträgern gegliedert und notariell beglaubigt mitgeteilt werden. Im Jahr 2005 entfiel der weitaus größte Teil der mit insgesamt zirka 18o Mio. Euro bezifferten Werbeausgaben der Tabakindustrie auf Promotion (zirka 86 Mio. Euro), gefolgt von Außenwerbung (zirka 52 Mio. Euro) [29]. Demgegenüber sind die Werbeausgaben für das Jahr 2006 beträchtlich auf knapp 79,9 Mio. Euro gesunken. Mehr als die Hälfte dieses Betrages (41,9 Mio. Euro) wurde für Promotion verwendet. An zweiter Stelle stehen die Ausgaben für Außenwerbung (20 Mio. Euro) [30]. Die Zahlen für das Jahr 2007 zeigen einen erneuten Anstieg der Werbeausgaben auf insgesamt 129 Mio. Euro. Das Werbeverbot in Printmedien und im Internet schlug sich deutlich in sehr viel geringeren Ausgaben für diese Bereiche nieder. Gleichzeitig stiegen 2007 die Ausgaben für Außenwerbung wieder auf fast das gleich hohe Niveau wie 2005 an, sodass sich auch die Gesamtsumme der Ausgaben im Vergleich zu 2006 wieder um mehr als 50\% erhöht hat [31].

\footnotetext{
3 Tabakerzeugnisse sind alle Erzeugnisse, die zum Rauchen, Schnupfen, Lutschen oder Kauen bestimmt sind, sofern sie ganz oder teilweise aus Tabak hergestellt sind (EU 2003).
} 


\section{Gesetzliche Vorschriften zur Regelung der Tabakwerbung}

Die Richtlinie 2003/33/EG ${ }^{4}$ des Europäischen Parlaments und des Rates vom 26. Mai 2003 zur Angleichung der Rechtsund Verwaltungsvorschriften der Mitgliedstaaten über Werbung und Sponsoring zugunsten von Tabakerzeugnissen regelt in Artikel 3 die Werbung in Druckerzeugnissen und Diensten der Informationsgesellschaft 5 , in Artikel 4 Rundfunkwerbung und Sponsoring und in Artikel 5 Sponsoring von Veranstaltungen [32]. Deutschland hat zunächst gegen die Richtlinie geklagt mit dem Ziel, Artikel 3 und 4 für nichtig erklären zu lassen. Nach Abweisung der Klage vom Europäischen Gerichtshof (EUGh) am 6. Dezember 2006 erfolgte die Umsetzung der EURichtlinie in nationales Recht in Deutschland im Dezember 2006. Seit 2005 sind Tabakerzeugnisse aus dem gesetzlichen Regelbereich für Lebensmittel herausgenommen und unterliegen den Anforderungen des "Vorläufigen Tabakgesetzes“ [33].

Tabakwerbung und -sponsoring in Rundfunk, Presse oder anderen Printmedien wurden mit $\$ \$ 21 a$ und 22 des „Vorläufigen Tabakgesetzes" [33] weitgehend untersagt. Ausnahmen bestehen für Veröffentlichungen, die sich ausschließlich an den Tabakhandel und an Raucher/innen wenden, und außerdem für Publikationen, die für den ausländischen Markt bestimmt sind (typisches Beispiel: Kataloge in Duty-Free-Shops). Verboten wurden mit der Neuregelung außerdem das Sponsoring von Rundfunkprogrammen und grenzüberschreitenden Veranstaltungen (Beispiel: Sponsoring von „Formel-1-Rennen") durch die Tabakindustrie. Auch das Verteilen von Probepackungen und Promotionsartikeln wurde untersagt. Darüber hinaus ist seither irreführende Werbung verboten, die zum

\footnotetext{
${ }^{4}$ ABI. EU Nr. L 152, S. 16, 2004 Nr. L 67, S. 34.

5 Art. 2 Abs. d der Richtlinie 2003/33/EG definiert Dienste der Informationsgesellschaft als „Dienste im Sinne des Art. 1 Abs. 2 der Richtlinie 98/34/EG", ABI. L 204 vom 21.7.1998, S. 37, geändert durch die Richtlinie 98/48/EG, ABI. L 217 vom 5.8.1998, S. 18 als: , jede in der Regel gegen Entgelt elektronisch im Fernabsatz und auf individuellen Abruf eines Empfängers erbrachte Dienstleistung".
}

Beispiel Rauchen als gesundheitlich unbedenklich darstellt, mit positivem Image verknüpft und deren Zielgruppe Kinder und Jugendliche sind.

Nach wie vor ist in Deutschland jedoch die Plakataußenwerbung und Kinowerbung ( $a b 18 \mathrm{Uhr}$ ) gestattet. Hier wird die Möglichkeit der EU-Richtlinie genutzt, Sponsoring und (indirekte) Werbung, die keine grenzüberschreitende Wirkung hat, auf nationaler Ebene so zu regeln, wie der jeweilige Mitgliedstaat dies zum Schutz der menschlichen Gesundheit für erforderlich hält. Außerdem stehen der Tabakindustrie trotz der beschriebenen Verbote zahlreiche indirekte Werbemethoden zur Verfügung. Diese reichen von Eventsponsoring (Sportveranstaltungen, Konzerte, Diskotheken) bis hin zum „Brand Stretching“6.

\section{Freiwillige Selbstbeschrän- kungsvereinbarungen mit der Tabakindustrie}

Seit dem Jahr 1972 liegen seitens des Verbandes der Cigarettenindustrie (VdC) als Interessenverband der Tabakfirmen in Deutschland Richtlinien über den Einsatz von Werbemitteln und -medien für die Werbung auf dem deutschen Markt vor, die ständig fortgeschrieben und zuletzt am 1. Juli 1996 modifiziert wurden. $\mathrm{Zu}$ diesen im Rahmen der Selbstverpflichtung formulierten Vereinbarungen gehörte unter anderem der Verzicht auf Plakataußenwerbung im Sichtbereich von 100 Metern von Schulen und Jugendzentren und auf Werbung in öffentlichen Verkehrsmitteln. Für die Einhaltung der Richtlinien beziehungsweise als Adressat für Meldungen von Verstößen wurde eine Schiedsstelle mit Sitz in Hamburg eingerichtet. Im Jahr 2007 löste sich der VdC auf und wurde 2008 mit teilweise veränderter Mitgliedschaft als Deutscher Zigarettenverband (DZV) neu gegründet. Gegenwärtig werden die freiwilligen Selbstbeschränkungen von den Mitgliedsunternehmen auch unter Berücksichtigung der veränderten gesetzlichen Rahmenbedingungen überarbeitet.

\footnotetext{
${ }^{6}$ Erweitern der Produktpalette um Nicht-TabakProdukte unter gleichem Markennamen, wie zum Beispiel Bekleidung („Marlboro-Classics") oder Kaffee („Benson \& Hedges").
}

\section{Basismaßnahme 3: Schutz vor Passivrauchen}

Die Gesundheitsgefährdung durch Passivrauchen ist belegt $[34,35,36,37,38]$. Die wirksamste Maßnahme zum Schutz von Nichtraucher(inne)n vor Passivrauchen ist eine rauchfreie Umwelt an Arbeitsplätzen, in Schulen, Krankenhäusern und gastronomischen Betrieben. Als eine Basismaßnahme des Gesundheitsziels „Tabakkonsum reduzieren“ wurde definiert, den Schutz vor Passivrauchen möglichst durch gesetzliche Regelungen $\mathrm{zu}$ erhöhen, sodass weniger Personen dem Passivrauchen ausgesetzt sind. Teilziele sind die Erhöhung der Zahl der rauchfreien öffentlich zugänglichen Einrichtungen, ein zunehmendes Bewusstsein der Schädlichkeit des Passivrauchens in Familien sowie eine breite Bekanntheit der gesundheitlichen Folgen des Passivrauchens.

Erfolgsindikatoren für den Schutz vor Passivrauchen und die damit verbundenen Maßnahmen sind auf der strukturellen Ebene eine Zunahme rauchfreier Bereiche in Krankenhäusern, Schulen und Betrieben sowie Rauchverbote in der Gastronomie. Der Nichtraucherschutz sollte gesetzlich geregelt sein. Auf der Bevölkerungsebene sind ein Rückgang der Prävalenz des Passivrauchens im privaten und öffentlichen Bereich und eine Zunahme des Bewusstseins für die Problematik der Gefährdung der Gesundheit durch Passivrauchen Erfolgsindikatoren [6].

Die Arbeitsstättenverordnung in der Fassung vom 3. Oktober 2002 garantiert für Arbeitsplätze ohne Publikumsverkehr rauchfreie Arbeitsplätze. Das Bundesgesetz zum Schutz vor den Gefahren des Passivrauchens vom 20. Juli 2007 sieht seit dem 1. September 2007 ein Rauchverbot in allen Einrichtungen des Bundes sowie der Verfassungsorgane des Bundes, in Verkehrsmitteln des öffentlichen Personenverkehrs und in Personenbahnhöfen vor. Die zwischen dem 1. August 2007 und dem 1. Juli 2008 in Kraft getretenen 16 Landesnichtraucherschutzgesetze sehen mit unterschiedlicher regionaler Ausgestaltung in Bezug auf Ausnahmeregelungen Rauchverbote in öffentlichen Einrichtungen der Länder, in Gesund- 


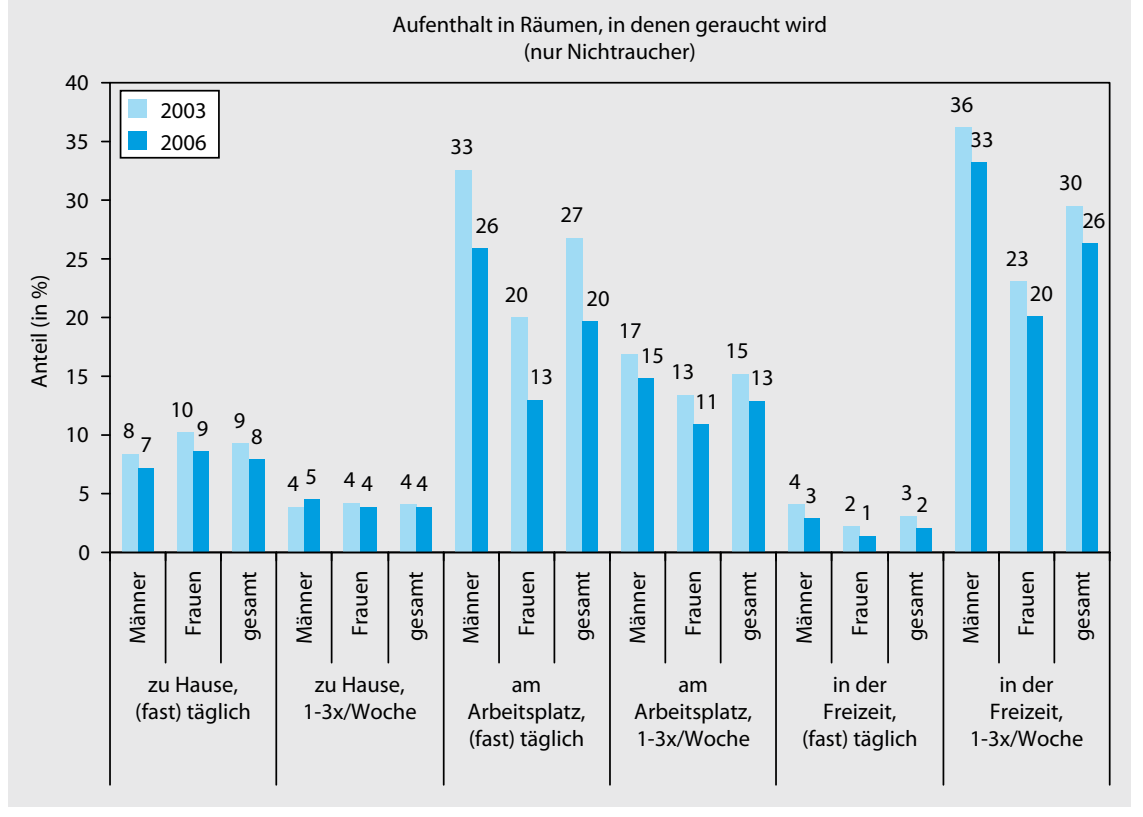

Abb. $1 \Delta$ Tabakrauchexposition von nicht rauchenden Erwachsenen nach Geschlecht und Expositionsort in den Jahren 2003 und 2006, Ergebnisse der Epidemiologischen Suchtsurveys 2003 und 2006. (Quelle: $[8,40]$ )

heits-, Kultur-, Sport- und Bildungseinrichtungen und in gastronomischen Betrieben vor.

Für die meisten dieser Indikatoren stehen mehrere Datenquellen zur Verfügung, darunter die Repräsentativerhebungen von BZgA, IFT und RKI. Aber auch die 13. Welle des Gesundheitsmonitors der Bertelsmann Stiftung, die Erwerbstätigenbefragung 2006 des Bundesinstituts für Berufsbildung und der Bundesanstalt für Arbeitsschutz und Arbeitsmedizin sowie Befragungen des DKFZ [39] liefern Informationen zur Prävalenz des Passivrauchens oder zu passivrauchbezogenen Einstellungen in der Bevölkerung.

\section{Ergebnisse}

Mit der Ermittlung der Passivrauchbelastung in den Jahren 2003 [40] und 2006 [8] erlaubt der Epidemiologische Suchtsurvey als einzige Datenquelle einen Zeitvergleich der Passivrauchexposition bei Erwachsenen. Die Daten zeigen, dass Erwerbstätige am Arbeitsplatz noch immer in erheblichem Ausmaß einer Passivrauchbelastung ausgesetzt sind (• Abb. 1). Männer sind am Arbeitsplatz und in der Freizeit in höherem MaBe mit Tabakrauch belastet als Frauen. Es zeigt sich aber auch, dass der Anteil der tabakrauchbelasteten Nichtraucher beiden Geschlechts sowohl zu Hause, am Arbeitsplatz als auch in der Freizeit im Zeitraum von 2003 bis 2006 zurückgegangen ist. Dabei fällt der Rückgang der Belastung am Arbeitsplatz am deutlichs-

Die Passivrauchexposition in verschiedenen Altersgruppen zeigen Ergebnisse des telefonischen Gesundheitssurveys 2006 [41]. Demnach ist der Anteil der Nichtraucher, die häufig einer Passivrauchbelastung ausgesetzt sind, in den jüngsten Altersgruppen unter 30 Jahren am höchsten und nimmt mit dem Alter ab. In allen Altersgruppen sind Männer etwas häufiger durch Tabakrauch belastet als Frauen. Die insbesondere am Arbeitsplatz und in der Freizeit hohe Passivgebnisse des Gesundheitsmonitors 2007 belegt [42]. Dass die Tabakrauchexposition am Arbeitsplatz nach Wirtschaftsbranche variiert und die Exposition bei männlichen und weiblichen Beschäftigten im Gastgewerbe am höchsten ist, zeigen Ergebnisse der Erwerbstätigenbefragung 2006 [39]. Hinsichtlich der Passivrauchexposition oder der Expositionshäufigkeit von Kindern und Jugendlichen ist es bislang nicht möglich, Trendaussagen zu treffen. Der Kinder- und Jugendgesundheitssurvey des RKI (KiGGS) ist ten aus. rauchbelastung wird außerdem durch Er- die bislang einzige Datenquelle zu dieser Thematik und wurde bis dato nur einmalig durchgeführt. Für den Erhebungszeitraum 2003 bis 2006 zeigen die Daten, dass sich von den befragten 11- bis 17-Jährigen etwa $85 \%$ der Jungen und $87 \%$ der Mädchen zumindest gelegentlich in Räumen aufhalten, in denen geraucht wird [41]. Je nach Altersgruppe sind zwischen einem Viertel und zwei Drittel der Jungen und Mädchen sogar mehrmals in der Woche einer Passivrauchbelastung ausgesetzt. Der Anteil der Jungen und Mädchen mit Passivrauchexposition nimmt mit dem Alter zu [41]. Darüber hinaus sind große soziale Unterschiede in der Altersgruppe 14 bis 17 Jahre festzustellen: Bei Hauptschüler/innen sind etwa dreimal so viele Jungen und Mädchen durch Passivrauchen belastet als bei Gymnasiasten/innen.

\section{Zunahme des Bewusstseins für die Problematik der Gefährdung der Gesundheit durch Passivrauchen}

Die Daten der BZgA-Repräsentativerhebungen "Förderung des Nichtrauchens“ aus den Jahren 2003 und 2005 erlauben einen Zeitvergleich der Einschätzung der Gesundheitsgefährlichkeit des Passivrauchens durch 12- bis 19-Jährige [12]. Die Anteile der Jugendlichen, die Passivrauchen als ziemlich oder sehr gesundheitsschädlich einordnen, sind gestiegen (von $56 \%$ auf $64 \%$ ), während die Anteile derer, die Passivrauchen als wenig oder gar nicht gesundheitsschädlich betrachten, zurückgegangen sind (von $12 \%$ auf $9 \%)$.

Auch die große Mehrheit der Erwachsenen stuft Passivrauchen als sehr gesundheitsgefährlich ein. Gemäß der International-Tobacco-Control-PolicyEvaluation- (ITC-)Deutschland-Befragung im Jahr 2007 [43] (eigene Berechnungen) schätzen $89 \%$ der Raucher/innen und 95\% der Nichtraucher/innen Zigarettenrauch als gefährlich für nicht rauchende Personen ein. Dass Passivrauchen Lungenkrebs verursachen kann, wissen $80 \%$ der Raucher/innen und $91 \%$ der Nichtraucher/innen. Über den Zusammenhang zwischen Passivrauchen und Asthma bei Kindern sind 86\% der Raucher/innen und $89 \%$ der Nichtraucher/ innen informiert. 
Der Anteil der nicht rauchenden Bevölkerung, der sich durch Raucher/innen gestört fühlt und der Orte meidet, an denen geraucht wird, lässt sich im Zeitvergleich mit Daten der Epidemiologischen Suchtsurveys 2003 und 2006 untersuchen $[8,40]$. Frauen fühlen sich häufiger stark belästigt als Männer (im Jahr 2006: 45\% der Frauen versus 37\% der Männer) und meiden auch häufiger Orte, an denen geraucht wird (63\% der Frauen versus 53\% der Männer). Im Zeitraum zwischen 2003 und 2006 hat sich in Bezug auf das Problembewusstsein nur wenig geändert, lediglich der Tendenz nach ist eine leichte Zunahme zu beobachten.

Die Unterstützung innerhalb der Bevölkerung ist ein wichtiger Maßstab für die Legitimation einer gesetzlichen Verankerung rauchfreier öffentlicher Innenräume sowie für die Einhaltung der gesetzlichen Regelungen. Die seit 2005 jährlich durchgeführten Bevölkerungsbefragungen des Deutschen Krebsforschungszentrums (DKFZ) zeigen, dass der Anteil derjenigen, die Rauchverbote in Gaststätten befürworten, von etwas mehr als der Hälfte der Befragten im Jahr 2005 auf fast drei Viertel der Befragten im Jahr 2009 angestiegen ist ( $\mathbf{A b b . ~ 2}$; [43]).

Die Ergebnisse des Gesundheitsmonitors 2007 belegen zudem, dass die Befürwortung von Rauchverboten sehr stark nach dem Ort oder Setting variiert, auf den/das sich das Rauchverbot beziehen soll [42]. So besteht in der Bevölkerung ein breiter gesellschaftlicher Konsens (mit einer Zustimmung von mehr als 75\%) über Rauchverbote an Arbeitsplätzen, in Restaurants, in öffentlichen Gebäuden sowie an Orten, an denen sich Kinder und Jugendliche aufhalten. Eine weniger breite Zustimmung zeigt sich für Rauchverbote in Kneipen und Bars (47\%) und an öffentlichen Plätzen (49\%). Die Zustimmungsquoten unterscheiden sich kaum nach Geschlecht.

\section{Basismaßnahme 4: Förderung des Ausstiegs aus der Tabakabhängigkeit}

Durch Beendigung des Rauchens lassen sich in jedem Alter ein Gesundheitsgewinn und eine Erhöhung der ferneren Lebenserwartung erzielen. Beispielswei-

Zustimmung zu Rauchverboten in der Gastronomie

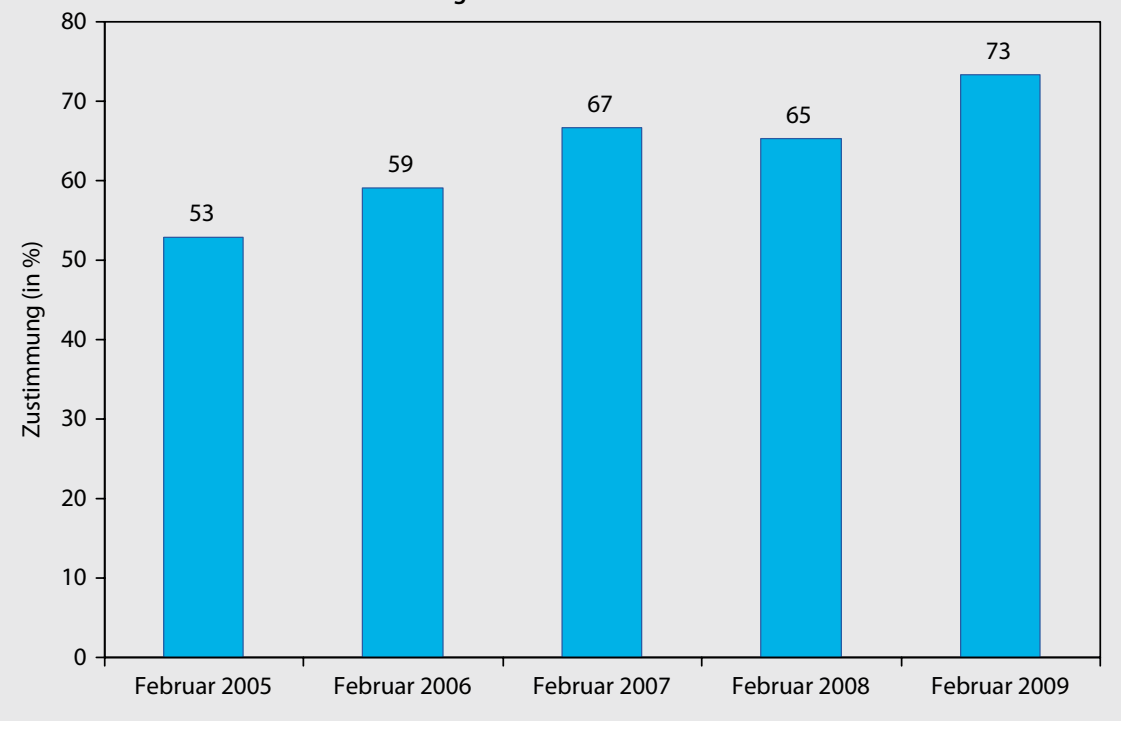

Abb. 2 A Anteil der Personen, die einem Rauchverbot in Gaststätten zustimmen, von 2005 bis 2009, Ergebnisse der vom DKFZ in Auftrag gegebenen GfK-Befragungen aus den Jahren 2005 bis 2009. (Quelle: [43])

se halbiert sich für Personen, die vor dem 50. Lebensjahr mit dem Rauchen aufhören, das Risiko, innerhalb der nächsten 15 Jahre zu versterben, im Vergleich zu Personen, die weiterrauchen [44]. Wenn Raucher vor ihrem 40. Lebensjahr mit dem Rauchen aufhören, erreichen sie wieder die statistische Lebenserwartung von Nierauchern [45]. Da nicht rauchende Menschen ein raucherfreundliches gesellschaftliches Klima mitbestimmen und als Modell zum Nachahmen verleiten, kann durch Beendigung des Rauchens ein langfristiger Trend zur Reduktion der Raucherquote in der Bevölkerung angestoßen werden. Durch Maßnahmen zur Förderung des Ausstiegs lassen sich langfristig deutlich höhere Abstinenzquoten erreichen als durch selbst initiierte Aufhörversuche [46]. Vor diesem Hintergrund wurde die „Förderung des Ausstiegs aus der Tabakabhängigkeit“ als Basismaßnahme beschrieben, für deren Erreichen folgende Maßnahmen empfohlen wurden:

- Bereitstellen von Beratungs- und Entwöhnungsangeboten unterschiedlicher Intensität (Kursangebote, Rauchertelefone, Kurzintervention, Internet, SMS), in unterschiedlichen Settings (am Arbeitsplatz, im Krankenhaus) und von unterschiedlichen Anbietern (zum Beispiel durch Tabakentwöhnungsspezialisten, durch
Krankenkassen, durch niedergelassene Ärzte und Ärztinnen),

- Streuen von Informationen (regelmäßig und breit) über die Schädlichkeit des Rauchens,

- Streuen von Informationen über das Vorhandensein von Beratungs- und Entwöhnungsangeboten,

- Motivieren der Bevölkerung zur Inanspruchnahme von Beratungs- und Entwöhnungsangeboten.

Erfolgsindikatoren sind (1) die Anzahl der bereitgestellten Beratungs- und Entwöhnungsangebote, (2) die Nachfrage nach diesen Angeboten, (3) die Inanspruchnahme dieser Beratungs- und Entwöhnungsangebote, (4) das Wissen bei Raucher(inne)n um Angebote zur Entwöhnung sowie (5) die Prävalenz der Aufhörwilligen beziehungsweise der Veränderungsbereitschaft in der rauchenden Bevölkerung. Aussagekräftigster Indikator ist (6) die Entwicklung der Aussteigerquote, also die Quote der Ex-Raucher/innen im Verhältnis zu den Personen, die jemals in ihrem Leben Raucher/innen waren (Jemals-Raucher/innen).

Datenquellen zur Messung der Erfolgsindikatoren für die Förderung des Ausstiegs aus der Tabakabhängigkeit sind Zählstatistiken von Anbietern von Beratungs- und Entwöhnungsmaßnahmen (Inanspruchnahme des Rauchertelefons 
der BZgA, Abgabestatistiken zum Rauchfrei Programm des IFT, Leistungsstatistik der Krankenkassen zur Inanspruchnahme von Leistungen nach $\$ 20$ SGB V zur Prävention von Suchterkrankungen, Vollerhebung des DKFZ und der BZgA zu Angeboten der Tabakentwöhnung in Deutschland) und die Prävalenzzahlen zu Ex-Rauchern in der Bevölkerung [Telefonischer Gesundheitssurvey (GSTel) [47, 48], Epidemiologischer Suchtsurvey (ESA) [8]].

\section{Ergebnisse}

\section{Anzahl der bereitgestellten Beratungs- und Entwöhnungsangebote}

Pro Jahr stehen Raucher(inne)n etwa 3000 Angebote zur Tabakentwöhnung mit etwa 250.00o Plätzen zur Verfügung stehen. $\mathrm{Zu}$ diesem Ergebnis kommt eine Recherche des DKFZ, die den Stand und die Struktur von Angeboten zur Tabakentwöhnung in Deutschland im Jahr 2007 beschreibt [49]. Dazu wurden über 9000 potenzielle Anbieter von ambulanter Tabakentwöhnung und Raucherberatung identifiziert und befragt. Das Ergebnis bedeutet, dass für weniger als $2 \%$ der Raucher/innen ein Beratungsangebot besteht. Es zeigten sich regionale Unterschiede: Vor allem in den neuen Bundesländern gibt es weniger Angebote als in den südlichen Bundesländern, in denen das Angebot am besten ist. Da diese Erhebung erstmals stattfand, kann keine Aussage darüber getroffen werden, wie sich das Angebot in den letzten Jahren verändert hat.

\section{Nachfrage von Beratungs- und Entwöhnungsangeboten}

In Deutschland stehen mehrere Beratungstelefone für Raucher/innen zur Verfügung. Die Inanspruchnahme der Telefonberatung der BZgA zum Thema „Rauchen und Nichtrauchen" ist von knapp 4000 Anrufen im Jahr 2005 auf über 11.00o im Jahr 2006 angestiegen. Im Jahr 2007 konnten knapp 9000 Gespräche gezählt werden [30]. Vermuteter Grund für den Anstieg sind die Warnhinweise auf den Zigarettenschachteln, die teilweise die Telefonnummer des Beratungstelefons ausweisen.
Kursangebote zur Vermeidung von Suchtmittelkonsum sind im Jahr 2007 gegenüber dem Jahr 2005 um 50\% von 10.131 Kursen auf 15.764 Kurse gestiegen. Diese Zahlen sind im Präventionsbericht über die Leistungen der Gesetzlichen Krankenversicherungen in der Primärprävention nach Paragraph 20, SGB V dargestellt ([50], S. 65). Der Bericht schlüsselt die Maßnahmen für das Handlungsfeld „Suchtmittelkonsum“ zwar nicht nach Stoffgruppen auf, jedoch haben 99,4\% aller Kursteilnehmerinnen und Kursteilnehmer in diesem Handlungsfeld einen Kurs zur Tabakentwöhnung in Anspruch genommen (persönliche Kommunikation, Volker Wanek, GKV-Spitzenverband). Von allen in Anspruch genommenen Präventionsmaßnahmen entfallen lediglich o,8\% auf das Handlungsfeld „Suchtmittelkonsum“. Da sich die Gesamtzahl aller Leistungen von 2006 auf 2007 um 29\% steigerte, ist der prozentuale Anteil des Handlungsfelds „Suchtmittelkonsum“ gegenüber dem Jahr 2005 sogar um o,1\% gesunken.

Die erhöhte Nachfrage und Inanspruchnahme von Entwöhnungsangeboten spiegelt sich wider in der Inanspruchnahme des deutschlandweit am weitesten verbreiteten Tabakentwöhnungsprogramms „Das Rauchfrei Programm“. Seit Einführung der Statistik im Jahr 1991 wurden im Jahr 2008 die meisten Programme abgegeben. Seit dem Jahr 2003 ist die Nachfrage deutlich gestiegen. Die Erhöhung der Zahl der bereitgestellten Kurse geht mit einer Zunahme von für das Programm geschulten Trainern einher. In den Jahren von 2003 bis 2008 wurden durchschnittlich 245 neue Trainer pro Jahr ausgebildet, gegenüber durchschnittlich 88 pro Jahr im Zeitraum von 1991 bis 2002 (unveröffentlichte Daten).

\section{Prävalenz der Aufhörwilligen beziehungsweise der Verän- derungsbereitschaft in der rauchenden Bevölkerung}

Laut ESA-Studie hat sich bei den aktuellen Raucher/innen die Bereitschaft, mit dem Rauchen aufzuhören, im Jahr 2006 im Vergleich zum Jahr 2003 nicht verändert. 54\% der Raucher/innen (gegenüber $55 \%$ im Jahr 2003) befanden sich im Stadium der Absichtslosigkeit, hatten also nicht vor, ihr Rauchverhalten in den nächsten sechs Monaten zu ändern, 40\% (gegenüber $41 \%$ ) gaben an, innerhalb der nächsten sechs Monate mit dem Rauchen aufhören zu wollen (Stadium der Absichtsbildung), und jeweils $5 \%$ gaben an, innerhalb der nächsten 30 Tage mit dem Rauchen aufhören zu wollen [8]. Auch bei der differenzierten Analyse der Bereitschaft nach Geschlecht und Alter zeigten sich keine bedeutsamen Veränderungen über die Zeit.

\section{Entwicklung der Aussteigerquote}

Die Aussteigerquote, das heißt der prozentuale Anteil von Ex-Raucher(inne)n an Jemals-Raucher(inne)n, schwankt bei den fünf Erhebungen von 1995 bis 2006 zwischen 38\% und 44\%. Im Jahr 2006 ist die Quote etwas angestiegen, nachdem sie in den 1990er-Jahren gesunken war (eigene Berechnungen aus Daten der ESA-Studie) (• Abb. 3).

Die Ergebnisse der telefonischen Gesundheitssurveys von 2003 und 2004 zeigen eine stabile Aussteigerquote von $46 \%$ bzw. 45,9\% für Männer und 44,3\% (identische Prozentzahl in beiden Erhebungen) für Frauen [47]. Die Prävalenz der Aussteiger liegt nach den Daten dieser Studie somit etwas höher als in der ESA-Studie. Beide Studien können keine Unterschiede zwischen Frauen und Männern ausmachen. Eine differenzierte Betrachtung der verschiedenen Altersgruppen zeigt einen deutlichen Anstieg der Aussteigerquote mit zunehmendem Alter (• Abb.4, [51], S. 13). Differenziert nach Alter werden auch Geschlechtsunterschiede deutlich. Die Aussteigerquote bei Frauen ist bis zum 40. Lebensjahr höher als bei den Männern, am deutlichsten in der Altersgruppe der 18- bis 29-Jährigen. Ab dem 50. Lebensjahr liegt die Aussteigerquote der Männer höher als bei den Frauen. Die Aussteigerquote in der Oberschicht liegt bei über $50 \%$ und ist damit um ein Viertel höher als bei Jemals-Raucher(inne)n aus der Unterschicht, von denen $38 \%$ aufgehört haben. Allgemein gilt: Je weniger geraucht wird, desto wahrscheinlicher ist es, dass ein/e Raucher/in mit dem Rauchen aufhört [47].

Die Anzahl der Raucher/innen, die in den letzten sechs Monaten mit dem Rauchen aufgehört haben, hat sich laut ESA- 
Studie im Jahr 2006 gegenüber dem Jahr 2003 von 4,9\% auf 8,1\% erhöht. Die Zunahme gilt für alle Altersgruppen und beide Geschlechter [8]. Mit zunehmendem Alter nimmt der Prozentsatz derjenigen $\mathrm{ab}$, die in den letzten sechs Monaten mit dem Rauchen aufgehört haben. Der Trend über die Jahre zeigt jedoch, dass der prozentuale Zuwachs an „frischen“ ExRaucher(inne)n mit etwa 50\% in der Altersgruppe der 18- bis 20-Jährigen und der über 40-Jährigen am größten ist.

\section{Basismaßnahme 5: Maßnahmen zur Verhinderung des Einstiegs in das Rauchen}

Die Maßnahmen zur Verhinderung des Einstiegs in das Rauchen konzentrieren sich auf Jugendliche, da dieser in der Regel in dieser Lebensphase stattfindet. Einrichtungen, in denen sich Kinder und Jugendliche befinden, sollen zunehmend rauchfreie Orte werden. Die schulische Prävention soll intensiviert und die Zahl frei zugänglicher Zigarettenautomaten verringert werden [6]. Weitere entscheidende Maßnahmen, die zur Verhinderung des Einstiegs beitragen, sind allgemeine Lebenskompetenzprogramme in Kindergärten und der Grundschule, suchtpräventive Lebenskompetenzprogramme in Grundschulen und der Sekundarstufe I, die Durchführung der Impulskampagne „Be Smart, Don’t Start“ für Schulklassen, personalkommunikative Maßnahmen wie die Mitmach-Parcours der BZgA, die Durchführung nationaler, massenmedialer Mehrebenenkampagnen zur Förderung des Nichtrauchens, Angebote an Eltern, die zum Beispiel deren Vorbildfunktion thematisieren, Lehrerfortbildungen, Rauchverbote in öffentlichen Einrichtungen, dem öffentlichen Personenverkehr und auf Bahnhöfen, Rauchbeschränkungen in Gaststätten, die Anhebung der Altersgrenze für die Abgabe von Tabakwaren an Jugendliche sowie technische Verkaufsbeschränkungen an Zigarettenautomaten für Minderjährige [52].

Indikatoren, mit denen der Erfolg dieser Basismaßnahme überprüft werden soll, sind unter anderem Änderungen der gesetzlichen Rahmenbedingungen, die Prävalenz des Rauchens sowie der Um-

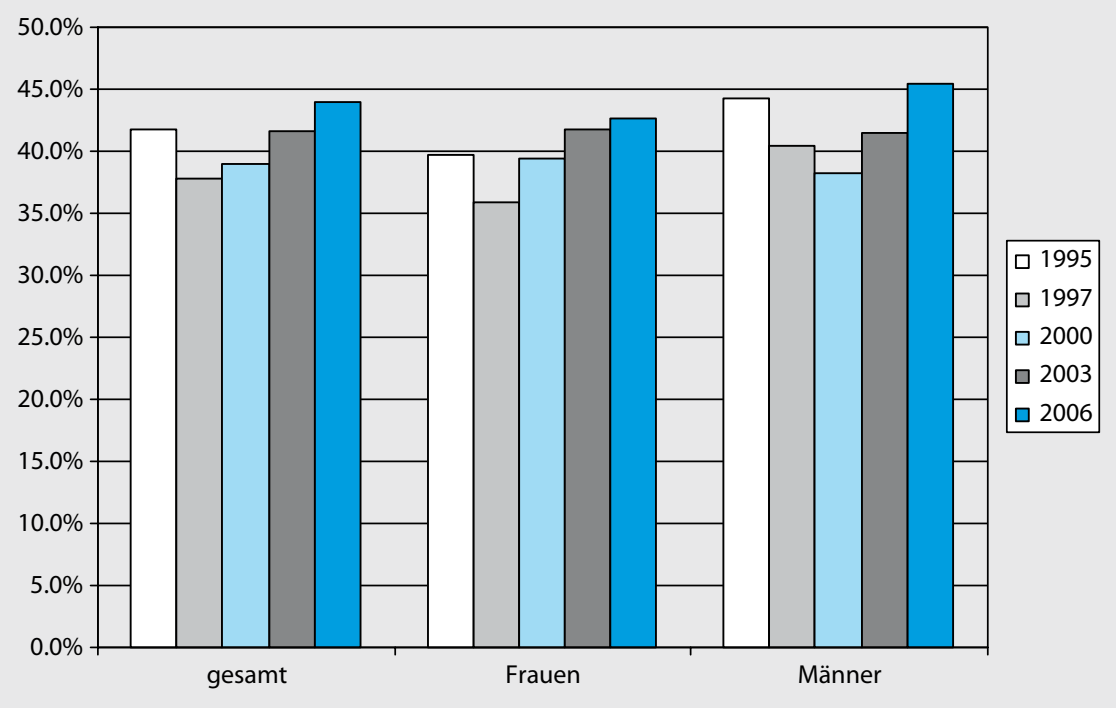

Abb. $3 \triangle$ Aussteigerquote: Verlauf über die Jahre (ESA-Daten). (Quelle: [8] und eigene Berechnungen)

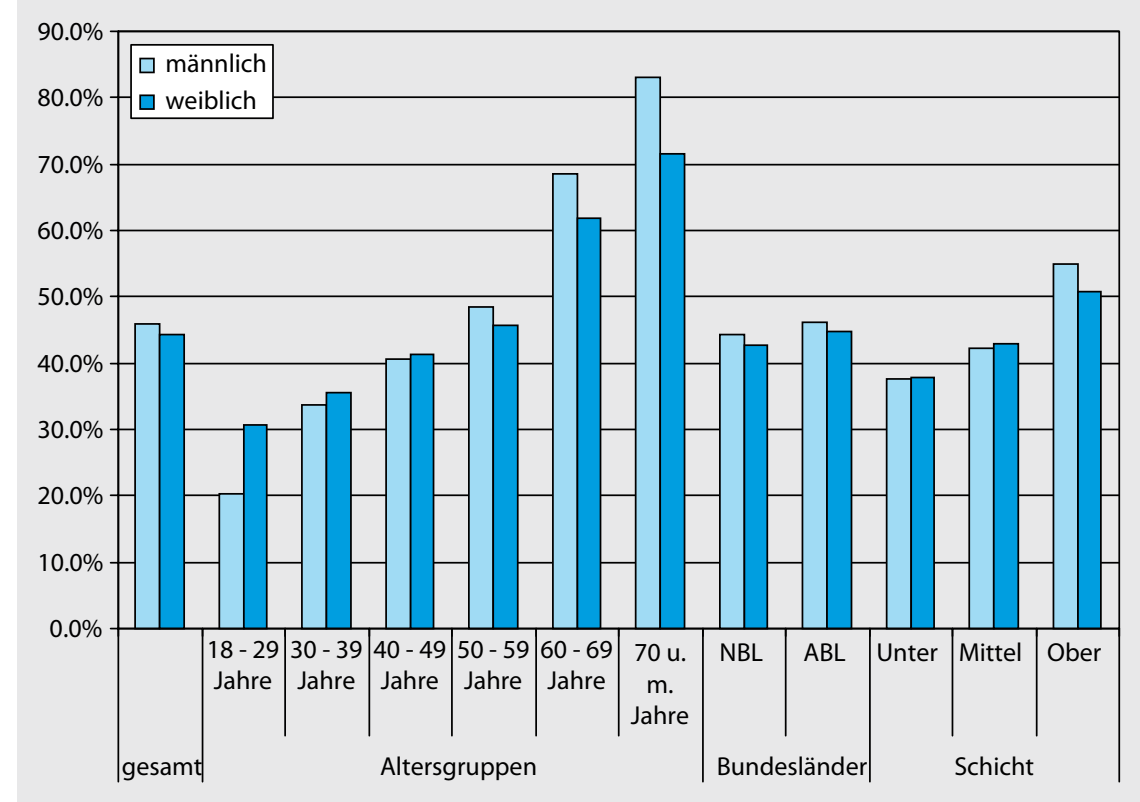

Abb. $4 \Delta$ Aussteigerquote, Unterschiede nach Geschlecht, Alter, Bundesländern und sozialer Schicht (GSTel-Daten). (Quelle: [47, 51])

fang von und die Teilnahme an schulischen und anderen individuumsbezogenen Präventivmaßnahmen zur Verhinderung des Einstiegs in das Rauchen [6].

Zur Bewertung struktureller Maßnahmen kann auf die Gesetzgebung der Bundesländer zu rauchfreien Schulen und andere Regelungen zurückgegriffen werden. Anhand der Repräsentativbefragungen der BZgA können die Wahrnehmung massenmedialer und die Teilnahme an schulischen Präventionsmaßnahmen sowie Einstellungsänderungen der Jugendlichen gegenüber dem Rauchen dargestellt werden. Aussagen zur Entwicklung des Anteils Jugendlicher, die noch nie geraucht haben, können die eingangs erwähnten repräsentativen Befragungen von Kindern, Jugendlichen und Schülerinnen und Schülern erbringen.

\section{Ergebnisse}

\section{Rauchfreie Schulen und andere strukturelle Veränderungen}

Im Juni 2004 war lediglich in Berlin ein Rauchverbot in Schulen in Kraft getreten. Die anderen Bundesländer folgten sukzessive. Mittlerweile gilt in allen Schulen Deutschlands ein Rauchverbot. 
Die öffentlich zugänglichen Zigarettenautomaten wurden mittlerweile auf Chipkarten umgerüstet. Damit wurde eine Verkaufssperre an Jugendliche unter 16 Jahren durchgesetzt. Seit dem 1. Januar 2008 gilt das Abgabeverbot für Tabakwaren an Jugendliche unter 18 Jahren, und am 1. Januar 2009 trat auch an Automaten das Abgabeverbot an unter 18-Jährige in Kraft. Die Zahl der Zigarettenautomaten ist in Deutschland von 835.000 im Jahr 2002 auf 470.000 im Jahr 2006 gesunken [52].

\section{Entwicklung des Anteils Jugendlicher, die noch nie geraucht haben}

Im Jahr 2001 erreichte der Anteil der 12bis 17-jährigen Jugendlichen, die noch nie geraucht haben, mit 40,5\% den Tiefpunkt innerhalb des gesamten Beobachtungszeitraums der BZgA-Studien [9]. Seitdem ist dieser Wert von Erhebung zu Erhebung kontinuierlich angestiegen und erreichte im Jahr 2008 mit 60,6\% den historisch höchsten Stand. Dieser deutliche Anstieg ist bei beiden Geschlechtern zu beobachten. Auch nach den ESPAD-Studien [13] hat der Anteil der Schülerinnen und Schüler im Alter von 15 bis 16 Jahren, die noch nie geraucht haben, zwischen 2003 und 2007 zugenommen.

\section{Zusammenfassende Bewertung des Evaluationsergebnisses}

Das Konzept des Gesundheitsziels „Tabakkonsum reduzieren“ wurde im Jahr 2003 entwickelt. Um die Empfehlungen für eine nachhaltige und erfolgreiche Tabakkontrollpolitik in Deutschland aktualisieren und an den derzeitigen Sachstand anpassen zu können, wurde mit diesem Beitrag eine Evaluation der Basismaßnahmen vorgenommen, die sich am Evaluationskonzept aus dem Jahr 2005 orientiert [6] und auf die bestehende Datenbasis in Deutschland zurückgreift.

Basismaßnahme 1 (Tabaksteuererhöhungen) forderte kontinuierliche Tabaksteuererhöhungen auf alle Tabakwaren. Die Steuererhöhungen zwischen 2002 und 2005 hatten eine deutliche Erhöhung des Zigarettenpreises zur Folge. Seit 2005 hat es allerdings keine Steuererhöhung mehr gegeben. Auch wenn infolge der Steuerer- höhungen bedeutende Anteile der Raucher/innen berichten, ihr Rauchverhalten verändert zu haben, ist ein Rückgang in der Rauchprävalenz nur bei Jugendlichen zu beobachten.

Ein vollständiges Verbot der direkten und indirekten Tabakwerbung, wie es Basismaßnahme 2 vorsieht, ist bislang nicht umgesetzt worden. Seit 2003 ist gemäß einer entsprechenden EU-Richtlinie Tabakwerbung und -sponsoring nun auch in Presse, Printmedien und Internet untersagt, außerdem wurde irreführende Werbung verboten. Da jedoch Plakataußenwerbungen und Kinowerbespots ( $\mathrm{ab}$ 18 Uhr) sowie Promotionen, Sponsoring und indirekte Werbung weiterhin erlaubt sind, zeigt sich eine Verlagerung der Werbeausgaben der Tabakindustrie in diese Bereiche.

Auch in Bezug auf Basismaßnahme 3 (Schutz vor Passivrauchen) wurden in den letzten Jahren große Schritte vollzogen, eine vollständige Erfüllung der Ziele ist aber nicht erfolgt. So sollen zwar die Nichtraucherschutzgesetze in Schulen, Gesundheitseinrichtungen und öffentlichen Einrichtungen eine vollständig rauchfreie Umwelt garantieren, die vielgestaltigen Ausnahmeregelungen insbesondere in der Gastronomie verhindern jedoch einen umfassenden Schutz. Erste Erfolge zeigen sich aber in der Bevölkerung in einem zunehmenden Problembewusstsein und einer zunehmenden Akzeptanz von Rauchverboten, auch ein Rückgang der Passivrauchprävalenz deutet sich an.

Hinsichtlich der Förderung des Ausstiegs aus dem Rauchen (Basismaßnahme 4) zeigt sich, dass sowohl die Angebote als auch die Nachfragen nach Entwöhnungsmaßnahmen in den letzten Jahren gestiegen sind. Inwieweit sich dies auch auf die Zahl an Rauchausstiegen auswirken wird, muss sich noch zeigen.

Zur Verhinderung des Einstiegs (Basismaßnahme 5) gab es in den letzten Jahren zahlreiche Maßnahmen, und zwar sowohl in Form struktureller als auch verhaltensorientierter Präventionsansätze. Die Erfolge schlagen sich bei Jugendlichen in einer sinkenden Raucherquote, einer steigenden Nieraucherquote und in einer zunehmend kritischeren Einstellung der Nieraucher/innen gegenüber dem Rauchen nieder.

\section{Empfehlungen}

In allen für das Gesundheitsziel „Tabakkonsum reduzieren“ identifizierten Maßnahmebereichen sind in den letzten Jahren Maßnahmen umgesetzt und Teilziele verfolgt worden. Doch ist der Zielerreichungsgrad für die einzelnen Teilziele unterschiedlich ausgeprägt und insgesamt noch steigerungsfähig. Auf allen Ebenen der Tabakprävention müssen die Anstrengungen weiter fortgeführt werden, das Potenzial insbesondere an strukturellen, aber auch massenmedialen und verhaltensbezogenen Maßnahmen ist noch nicht ausgeschöpft.

Zunächst sind weitere Erhöhungen der Tabaksteuer und eine Angleichung der Besteuerung von Feinschnitt zu empfehlen. Auch an der Forderung eines umfassenden Werbeverbots ist weiterhin festzuhalten. Hinsichtlich einer rauchfreien Umwelt sollte ein generelles Rauchverbot an Arbeitsplätzen über die Arbeitsstättenverordnung realisiert werden, mindestens jedoch ein bundesweit einheitliches und umfassendes Rauchverbot in der Gastronomie. Zur Förderung der Nachfrage nach Tabakentwöhnungsmaßnahmen ist eine höhere diesbezügliche Kostenerstattung zu diskutieren.

Neben diesen strukturellen Maßnahmen sollte gleichzeitig eine auf Erwachsene zugeschnittene Aufklärungskampagne über die Risiken des Passivrauchens, des Rauchens und die Möglichkeiten der Entwöhnung ausgebaut werden. Bei der Entwicklung und Implementierung von Entwöhnungsangeboten sollte auch berücksichtigt werden, inwieweit die Teilnahme und der Entwöhnungserfolg durch die soziale Lage der Interessenten mitbestimmt werden. Im Bereich der Tabakentwöhnung muss die Vernetzung zwischen den Akteuren im Gesundheitswesen und den Entwöhnungsexperten verbessert werden. Auch muss das Engagement von Ärzt(inn)en gefördert werden, rauchende Patient(inn)en zum Rauchstopp zu motivieren und darin zu unterstützen. Das Angebot an Raucherentwöhnungskursen durch Krankenkassen ist auszuweiten. Gefordert sind zielgruppenspezifische Programme, insbesondere für Schwangere und Beschäftigte im Gesundheitswesen. Die volle Übernahme der Kosten 
unter anderem auch von Nikotinpräparaten zum Beispiel im Rahmen von Disease-Management-Programmen durch die Kostenträger sollte geprüft werden.

Zur Verhinderung des Einstiegs ins Rauchen sollten die massenmedialen Maßnahmen für Jugendliche intensiviert und verstärkt durch personalkommunikative Angebote ergänzt werden.

Um die ersten ermutigenden Erfolge nachhaltig zu sichern und zukünftig zu verbessern, sollten die aus den Ergebnissen der Evaluation herausgearbeiteten Empfehlungen im Rahmen einer umfassenden und evidenzbasierten Präventionsstrategie umgesetzt werden. Der Kooperationsverbund gesundheitsziele.de will hierzu einen Beitrag leisten. Auf Basis der aktuellen wissenschaftlichen Evidenz werden die Teilziele neu justiert und mit geeigneten Umsetzungsmaßnahmen unterlegt. Das so aktualisierte Gesamtkonzept wird der Politik und den Akteur/innen im Gesundheitswesen als Empfehlung vorgelegt und soll ihnen als Orientierung für ihr Handeln dienen. Auf dieser Grundlage kann die Nachsteuerung des Gesundheitsziels erfolgen, das heißt, die Teilziele werden an die veränderte Ausgangslage angepasst, und es werden neue Akzente für ihre Umsetzung gesetzt.

\section{Hinweis}

Frau U. Mons wird von der Klaus Tschira Stiftung, gemeinnützige Gesellschaft mit beschränkter Haftung. gefördert.

\section{Korrespondenzadresse}

\section{Prof. Dr. U. Maschewsky-Schneider}

Berlin School of Public Health an der Charité,

Charité - Universitätsmedizin Berlin

Oudenarder Str 16, 13347 Berlin

ulrike.maschewsky-schneider@charite.de

Interessenkonflikt. Der korrespondierende Autor gibt an, dass kein Interessenkonflikt besteht.

\section{Literatur}

1. Weltgesundheitsorganisation (WHO) (2008) 10 Facts on the tobacco epidemic and global tobacco control. http://www.who.int/features/ factfiles/tobacco_epidemic/tobacco_epidemic_ facts/en/index.html

2. Bundesministerium für Gesundheit (BMG) (Hrsg) (2003) gesundheitsziele.de - Forum zur Entwicklung und Umsetzung von Gesundheitszielen in Deutschland. Bericht. Tabakkonsum reduzieren. Berlin, S 91-130
3. Pott E, Lang P, Töppich J (2003) Gesundheitsziel: Tabakkonsum reduzieren. Bundesgesundheitsbl Gesundheitsforsch Gesundheitsschutz 46:150155

4. Gesellschaft für Versicherungswissenschaft und -gestaltung (GVG), gesundheitsziele.de (2004) Basismaßnahmen zur Erreichung des Gesundheitsziels „Tabakkonsum reduzieren“. Köln

5. Drogen- und Suchtrat (9. Juni 2008) Empfehlungen des Drogen- und Suchtrates an die Drogenbeauftragte der Bundesregierung für ein Nationales Aktionsprogramm zur Tabakprävention. http://www.bmg.bund.de/cln_110/SharedDocs/Downloads/DE/Standardartikel/D/ Glossar-Drogenbeauftragte/Tabak_Aktionsplan ,templateld=raw,property=publicationFile.pdf/ Tabak_Aktionsplan.pdf

6. Maschewsky-Schneider U, Lampert T, Kröger C et al (2006) Evaluation des Gesundheitsziels „Tabakkonsum reduzieren“. Bundesgesundheitsbl Gesundheitsforsch Gesundheitsschutz 49:11551161

7. Lampert T, Thamm M (2008) Tabak - Zahlen und Fakten zum Konsum. In: Deutsche Hauptstelle für Suchtfragen (Hrsg) Jahrbuch Sucht 2008. Neuland, Geesthacht, S 54-72

8. Baumeister SE, Kraus L, Stonner TK, Metz K (2008) Tabakkonsum, Nikotinabhängigkeit und Trends. Ergebnisse des Epidemiologischen Suchtsurveys 2006. Sucht 54 (Sonderheft 1):2635

9. Bundeszentrale für gesundheitliche Aufklärung (in Vorbereitung) Die Drogenaffinität Jugendlicher in der Bundesrepublik Deutschland 2008. Eine Wiederholungsbefragung der Bundeszentrale für gesundheitliche Aufklärung. Verbreitung des Tabakkonsums bei Jugendlichen und jungen Erwachsenen. BZgA, Köln

10. Bundeszentrale für gesundheitliche Aufklärung (2008) Die Drogenaffinität Jugendlicher in der Bundesrepublik Deutschland 2008. Alkohol-, Tabak- und Cannabiskonsum. Erste Ergebnisse zu aktuellen Entwicklungen und Trends. BZgA, Köln

11. Bundeszentrale für gesundheitliche Aufklärung (2007) Förderung des Nichtrauchens bei Jugendlichen 2007. Eine Wiederholungsbefragung der Bundeszentrale für gesundheitliche Aufklärung. Kurzbericht. BZgA, Köln

12. Bundeszentrale für gesundheitliche Aufklärung (2006) Förderung des Nichtrauchens. Eine Wiederholungsbefragung der Bundeszentrale für gesundheitliche Aufklärung. BZgA, Köln

13. Kraus L, Papst A, Steiner S (2008) Europäische Schülerstudie zu Alkohol und anderen Drogen 2007 (ESPAD). Befragung von Schülerinnen und Schülern der 9. und 10. Klasse in Bayern, Berlin, Brandenburg, Hessen, Mecklenburg-Vorpommern, Saarland und Thüringen (IFT-Berichte Bd. 165). IFT, München

14. Richter M, Leppin A (2008) Trends im Tabak-, Alkohol- und Cannabiskonsum im frühen Jugendalter. In: Deutsche Hauptstelle für Suchtfragen (Hrsg) Jahrbuch Sucht 08. Neuland, Geesthacht, S 152-170

15. Kurth B-M (2007) Der Kinder- und Jugendgesundheitssurvey (KiGGS): Ein Überblick über Planung, Durchführung und Ergebnisse unter Berücksichtigung von Aspekten eines Qualitäts managements. Bundesgesundheitsbl Gesundheitsforsch Gesundheitsschutz 50:533-546
16. Lampert T, List SM (2009) Tabak - Zahlen und Fakten zum Konsum. In: Deutsche Hauptstelle für Suchtfragen e.V. (Hrsg) Jahrbuch Sucht 2009. Neuland, Geesthacht, S 51-71

17. gesundheitsziele.de. (2004) Basismaßnahmen zur Erreichung des Gesundheitsziels „Tabakkonsum reduzieren". Arbeitspapier der AG 6 Tabakkonsum reduzieren. Verabschiedet vom gesundheitsziele.de-Ausschuss am 6. Juli 2004. http//www.gesundheitsziele.de

18. Lampert T (2008) Daten zum Rauchen in Deutschland. Neue Entwicklungen im Rauchverhalten von Erwachsenen. Vortrag anlässlich der 6. Deutschen Konferenz für Tabakkontrolle, Heidelberg

19. Hanewinkel R, Isensee B (2002) Umsetzung, Akzeptanz und Auswirkungen der Tabaksteuererhöhung vom 1. Januar 2002. Bevölkerungsrepräsentative Untersuchung im Auftrag des Bundesministeriums für Gesundheit. Abschlussbericht, Kiel

20. Hanewinkel R, Isensee B (2004) Umsetzung, Akzeptanz und Auswirkungen der Tabaksteuererhöhung vom 1. März 2004. Bevölkerungsrepräsentative Untersuchung im Auftrag des Bundesministeriums für Gesundheit und Soziale Sicherung. Kiel

21. Hanewinkel R, Isensee B (2005) Umsetzung, Akzeptanz und Auswirkungen der Tabaksteuererhöhung vom 1. Dezember 2004. Untersuchung im Auftrag des Bundesministeriums für Gesundheit. Kiel

22. Plamper E, Klever Deichert G, Lauterbach KW (2006) Auswirkungen der Tabaksteuererhöhungen in Deutschland auf den Tabakkonsum und Konsequenzen für die Gesundheitspolitik. Bundesgesundheitsbl Gesundheitsforsch Gesundheitsschutz 49:660-664

23. Lovato C, Linn G, Stead LF, Best A (2003) Impact of tobacco advertising and promotion on increasing adolescent smoking behaviours. Cochrane Database Syst Rev:CD003439

24. Paynter J, Edwards R (2009) The impact of tobacco promotion at the point of sale: a systematic review. Nicotine Tob Res 11(1):25-35

25. World Bank (2003) Der Tabakepidemie Einhalt gebieten: Regierungen und wirtschaftliche Aspekte der Tabakkontrolle. Hrsg. der deutschen Ausgabe für die World Bank: Dtsch Krebsforschungszentrum. Heidelberg

26. Hanewinkel R, Sargent JD (2007) Rauchen in Film und Fernsehen. Wirkungen auf Kinder und Jugendliche. IFT, Kiel http://www.bmg.bund.de/ cln_110/nn_1196876/SharedDocs/Downloads/ DE/Neu/Tabak_Rauchverhalten_20Kinder,templ ateld=raw, property=publicationFile.pdf/Tabak_ Rauchverhalten\%20Kinder.pdf

27. gesundheitsziele.de (2005) Daten und Indikatoren zur Evaluierung des Gesundheitsziels „Tabakkonsum reduzieren". Berlin

28. Weltgesundheitsorganisation (WHO) (2004) Rahmenübereinkommen der WHO zur Eindämmung des Tabakgebrauchs. Amtliche deutsche Übersetzung vom 2. April 2004

29. Drogenbeauftragte der Bundesregierung, Bundesministerium für Gesundheit (Hrsg) (2007) Drogen- und Suchtbericht 2006. Berlin

30. Drogenbeauftragte der Bundesregierung, Bundesministerium für Gesundheit (Hrsg) (2008) Drogen- und Suchtbericht 2008. Berlin 
31. Drogenbeauftragte der Bundesregierung. Bundesministerium für Gesundheit (2009) Drogenund Suchbericht 2009. Berlin

32. Europäische Union (2003) Richtlinie 2003/33/ EG des Europäischen Parlaments und des Rates vom 26. Mai 2003 zur Angleichung der Rechtsund Verwaltungsvorschriften der Mitgliedsstaaten über Werbung und Sponsoring zugunsten von Tabakerzeugnissen. Amtsblatt der Europäischen Union L 152/16. Brüssel

33. Bundesministerium der Justiz (BMJ) (2006) Vorläufiges Tabakgesetz in der Fassung der Bekanntmachung vom 9. September 1997 (BGBI. I S. 2296), zuletzt geändert durch Artikel 1 des Gesetzes vom 21. Dezember 2006 (BGBI. I S 3365). Berlin. http://www.bundesrecht.juris.de/ Img_1974/

34. International Agency for Research on Cancer (2004) Tobacco smoke and involuntary smoking. IARC Monographs on the evaluation of the carcinogenic risks to humans. International Agency for Research on Cancer and World Health Organisation. Vol. 83. Lyon, International Agency for Research on Cancer, World Health Organization

35. Jöckel KH (2000) Gesundheitsrisiken durch Passivrauchen. Dtsch Ärztebl 97:A2852-A2857

36. Wichmann HE, Jöckel KH, Becher H (1999) Gesundheitliche Risiken durch Passivrauchen - Bewertung der epidemiologischen Daten. Umweltmed Forschung Praxis 4:28-42

37. Deutsches Krebsforschungszentrum (2005) Passivrauchen - ein unterschätztes Gesundheitsrisiko. DKFZ, Heidelberg

38. Janson C, Chinn S, Jarvis D et al (2001) Effect of passive smoking on respiratory symptoms, bronchial responsiveness, lung function, and total serum IgE in the European Community Respiratory Health Survey: a cross-sectional study. Lancet 358:2103-2109

39. Deutsches Krebsforschungszentrum (2007) Erhöhtes Gesundheitsrisiko für Beschäftigte in der Gastronomie durch Passivrauchen am Arbeitsplatz. DKFZ, Heidelberg

40. Augustin R, Metz K, Heppekausen K, Kraus L (2005) Tabakkonsum, Abhängigkeit und Änderungsbereitschaft. Ergebnisse des Epidemiologischen Suchtsurvey 2003. Sucht 51 Sonderheft 1:40-48

41. Lampert T (2008) Tabakkonsum und Passivrauchbelastung von Jugendlichen, Dtsch Ärztebl 105:265-271

42. Mons U, Amhof R, Pötschke-Langer M (2008) Gesetzliche Maßnahmen zum Nichtraucherschutz in Deutschland - Einstellungen und Akzeptanz in der Bevölkerung. In: Böcken J, Braun B, Amhof R (Hrsg) Gesundheitsmonitor 2008. Gesundheitsversorgung und Gestaltungsoptionen aus der Perspektive der Bevölkerung. Bertelsmann Stiftung, Gütersloh, S 181-209

43. Deutsches Krebsforschungszentrum (2009) Rauchfreie Gaststätten in Deutschland 2009: Steigende Zustimmung in der Bevölkerung. DKFZ, Heidelberg

44. DHHS (U.S. Department of Health and Human Services) (1990) The Health Benefits of Smoking Cessation. DHHS Publication No. (CDC) 90-8416

45. Doll R, Peto R, Boreham J, Sutherland I (2004) Mortality in relation to smoking: 50 years observations on male British doctors. BMJ 328:1519-1527
46. Fiore MC, Bailey WC, Cohen SJ et al (2008) Treating tobacco use and dependence: 2008 Update - Clinical Practice Guideline. U.S. Department of Health and Human Services, U.S. Public Health Service, Rockville, MD

47. Lampert T, Burger M (2005) Verbreitung und Strukturen des Tabakkonsums in Deutschland. Bundesgesundheitsbl Gesundheitsforsch Gesundheitsschutz 48:1231-1241

48. Lampert T, Burger M (2004) Rauchgewohnheiten in Deutschland - Ergebnisse des telefonischen Bundes-Gesundheitssurveys 2003. Gesundheitswesen 66:511-517

49. Etzel M, Mons U, Schmitt S et al (2008) Raucherentwöhnung in Deutschland 2007 - Struktur der ambulanten Therapieangebote zur Tabakentwöhnung und Raucherberatung. Bundesgesundheitsbl Gesundheitsforsch Gesundheitsschutz

50. Medizinischer Dienst des Spitzenverbandes Bund der Krankenkassen e.V. (MDS) (Hrsg) (2008) Präventionsbericht 2008, Dokumentation von Leistungen der gesetzlichen Krankenversicherungen in der Primärprävention und betrieblichen Gesundheitsförderung. Essen. http://www.mds-ev.de

51. Ellert U, Wirz J, Ziese T (2006) Beiträge zur Gesundheitsberichterstattung des Bundes. Telefonischer Gesundheitssurvey des Robert KochInstituts (2. Welle). Deskriptiver Ergebnisbericht. Robert Koch-Institut, Berlin

52. Facharbeitsgruppe "Suchtprävention“ im Auftrag des Drogen und Suchtrats (2008) Empfehlungen des Drogen- und Suchtrates an die Drogenbeauftragte der Bundesregierung für ein Nationales Aktionsprogramm zur Tabakprävention. Untersuchung im Auftrag des Bundesministeriums für Gesundheit. Kiel

\section{Hellmich \\ Qualitätsmanagement und Zertifizierung im Rettungsdienst} Grundlagen - Techniken -

Modelle - Umsetzung

Berlin, Heidelberg: Springer Verlag 2009, 1., 224 S., 67 Abb., (ISBN 978-3-642-02169-5), gebunden, 49.95 EUR

Die Einführung von Qualitätsmanagement-Systemen im Rettungsdienst hat vor circa 10 Jahren begonnen. Mittlerweile kommt kaum eine Hilfsorganisation oder ein privater Betreiber an einer Einführung und Zertifizierung vorbei. Sich mit dieser Thematik auseinanderzusetzen, ist aber nicht immer einfach. Eine ganze Menge von Systemen und Möglichkeiten bieten sich hier an. Und die häufig hinzugezogenen Beratungsunternehmen bringen allzu oft auch kein Licht ins Dunkel.

Dem kann nun abgeholfen werden. In dem vorliegenden Buch wird das Thema Qualitätsmanagement von allen Seiten beleuchtet und das mit dem speziellen Fokus auf den Rettungsdienst.

Nach einigen Grundsätzen zu Qualität, Qualitätsmanagement und Qualitätspolitik beleuchtet der Autor die Qualitätsplanung sowie die Implementierung und Umsetzung. Ein weiteres Kapitel widmet sich dem Prozess der kontinuierlichen Verbesserung. In diesem Abschnitt werden verschiedene Methoden wie das Kai Zen, Fehlermanagement und das Benchmarking beschrieben. Abgerundet wird das Buch mit einer Beschreibung der verschiedensten Systeme. Neben der eigentlichen ISO 9000 ff finden sich hier ebenfalls Informationen zum EFQM Modell, Six Sigma und vielen weiteren. In allen Kapiteln wird der Bezug zum Rettungsdienst hergestellt, was für den Leser besonders nutzbringend ist. Neben dem eigentlichen Grundlagenwissen werden viele Hintergrundinformationen sowie Tipps zur Einführung und Umsetzung eines Qualitätsmanagement-System vermittelt.

Der Autor schafft es, dieses mitunter sehr trockene Thema locker zu vermitteln und dabei doch seriös und verständlich zu bleiben. Jeder, der sich ansatzweise mit dem Thema beschäftigen muss oder will, kommt an diesem Buch nicht vorbei. Es sollte zur Standardliteratur jeder Rettungswachen-Bibliothek und jedes QM-Beauftragten im Rettungsdienst gehören.

Frank Flake

(Qualitätsbeauftragter und stv. Leiter Rettungsdienst, MHD Bundesebene) 